SHORT REPORT

\title{
Activation induced cytidine deaminase expression in lymphocyte predominant Hodgkin lymphoma
}

\section{A Mottok, M-L Hansmann, A Bräuninger}

J Clin Pathol 2005;58:1002-1004. doi: 10.1136/icp.2005.026252

Background: The lymphocytic and histiocytic (L\&H) cells of lymphocyte predominant Hodgkin lymphoma $(\mathrm{HL})$ originate from germinal centre $B$ cells and carry mutated $V$ gene rearrangements, usually with intraclonal diversity. It is unclear whether intraclonal $V$ gene diversification by somatic hypermutation, which is strictly dependent on the enzyme activation induced cytidine deaminase (AID), is restricted to the early phase of lymphoma clone expansion and later silenced, or whether it remains active throughout malignant proliferation.

Aims: To analyse whether AID is expressed in L\&H cells as an indicator of active somatic hypermutation in the tumour cells. Methods: L\&H cells from lymphocyte predominant $\mathrm{HL}$ cases and centroblasts from lymphadenites were micromanipulated and analysed for AID expression by quantitative real time polymerase chain reaction.

Results: The AID transcription level was higher than background in three of the six lymphocyte predominant $\mathrm{HL}$ cases, although it was lower than that seen in centroblasts.

Conclusions: Somatic hypermutation may remain active in L\&H cells in a considerable proportion of cases, increasing the risk of acquiring further transforming mutations.

$\mathrm{H}$ odgkin lymphoma (HL) is subdivided into classic forms and lymphocyte predominant HL. The lymphocytic and histiocytic (L\&H) cells of lymphocyte predominant HL express B cell markers and also the germinal centre (GC) marker BCL6, indicating that they are derived from GC B cells. ${ }^{1}$ Analysis of V gene rearrangements in single L\&H cells revealed the presence of somatic mutations with intraclonal diversity in functional monoclonal $\mathrm{V}$ gene rearrangements, in line with a GC B cell origin..$^{2-5}$

\section{"It is unclear whether intraclonal V gene diversification by somatic hypermutation is restricted to the early phase of lymphoma clone expansion, and later silenced, or whether it remains active throughout malignant proliferation of the lymphoma cells"}

Somatic hypermutation requires activation induced cytidine deaminase (AID). ${ }^{6}$ Recent studies of AID protein expression using western blot analysis showed that AID expression is largely restricted to normal and transformed GC B cells and correlates well with the expression of AID transcripts. ${ }^{7}$ Several studies analysing AID expression and mutation patterns in $\mathrm{V}$ gene rearrangements in various $\mathrm{B}$ cell non-HLs showed that, although somatic hypermutation activity is strictly dependent on AID, the expression of this protein does not necessarily indicate ongoing somatic hypermutation..$^{7-10}$
Intraclonal diversity in $\mathrm{V}$ gene rearrangements as a result of active somatic hypermutation is a typical feature of GC B cells. However, for lymphomas showing intraclonal diversity it is unclear whether intraclonal $\mathrm{V}$ gene diversification by somatic hypermutation is restricted to the early phase of lymphoma clone expansion, and later silenced, or whether it remains active throughout malignant proliferation of the lymphoma cells. ${ }^{11}$ To clarify this issue for lymphocyte predominant HL we quantitatively examined the expression of AID in the L\&H cells of six patients with lymphocyte predominant HL at the transcription level, because suitable antibodies for immunohistochemistry are not yet available.

\section{MATERIALS AND METHODS}

\section{Micromanipulation of cells}

Lymph node biopsies of six patients with lymphocyte predominant HL and three with non-specific lymphadenitis were analysed. Frozen sections ( $5 \mu \mathrm{m}$ thick) were mounted on membrane covered slides (PALM, Bernried, Germany) and stained for four minutes with haematoxylin containing $200 \mathrm{U} / \mathrm{ml}$ RNase inhibitor (Roche, Mannheim, Germany). Sections were washed in water for two minutes, incubated with $2 \%$ eosin for 20 seconds, washed again, and then dried for three hours at $37^{\circ} \mathrm{C}$.

For each sample, single L\&H cells were microdissected and 50 of them were catapulted into one $25 \mu \mathrm{l}$ aliquot of Purescript lysis buffer (Biozym, Hamburg, Germany) using an ultraviolet laser beam (PALM microdissection system with a Zeiss Axiovert 200M microscope). From the same sections, 250 endothelial cells in groups of five were pooled together as a negative control sample. Fifty centroblasts from each of three cases of lymphadenitis, micromanipulated in groups of five cells, were used as positive control samples. We analysed four samples and four control samples from each case.

\section{Quantitative real time PCR}

Total RNA was isolated with the Purescript RNA isolation kit (Biozym), reducing all volumes to one tenth of the standard protocol, and adding glycogen as a carrier. Reverse transcription (RT) was performed using the First Strand cDNA synthesis kit (Roche), with a quarter of the standard volumes and the complete RNA isolated from one sample in a total volume of $5 \mu \mathrm{l}$. Quantitative real time polymerase chain reaction (PCR) was performed on an ABI PRISM 7900HT sequence detection system using Assays-on-Demand (Applied Biosystems, Darmstadt, Germany) for both AID and the 18S rRNA endogenous control (catalogue numbers Hs00221068_ml and 4319413E, respectively; Applied Biosystems) and the complete First Strand reaction for each PCR.

Abbreviations: AID, activation induced cytidine deaminase; $\mathrm{Ct}$, threshold cycle; GC, germinal centre; HL, Hodgkin lymphoma; L\&H, lymphocytic and histiocytic; PCR, polymerase chain reaction; RT, reverse transcription 
Table 1 Analysis of $\mathrm{V}$ gene rearrangements of micromanipulated L\&H cells

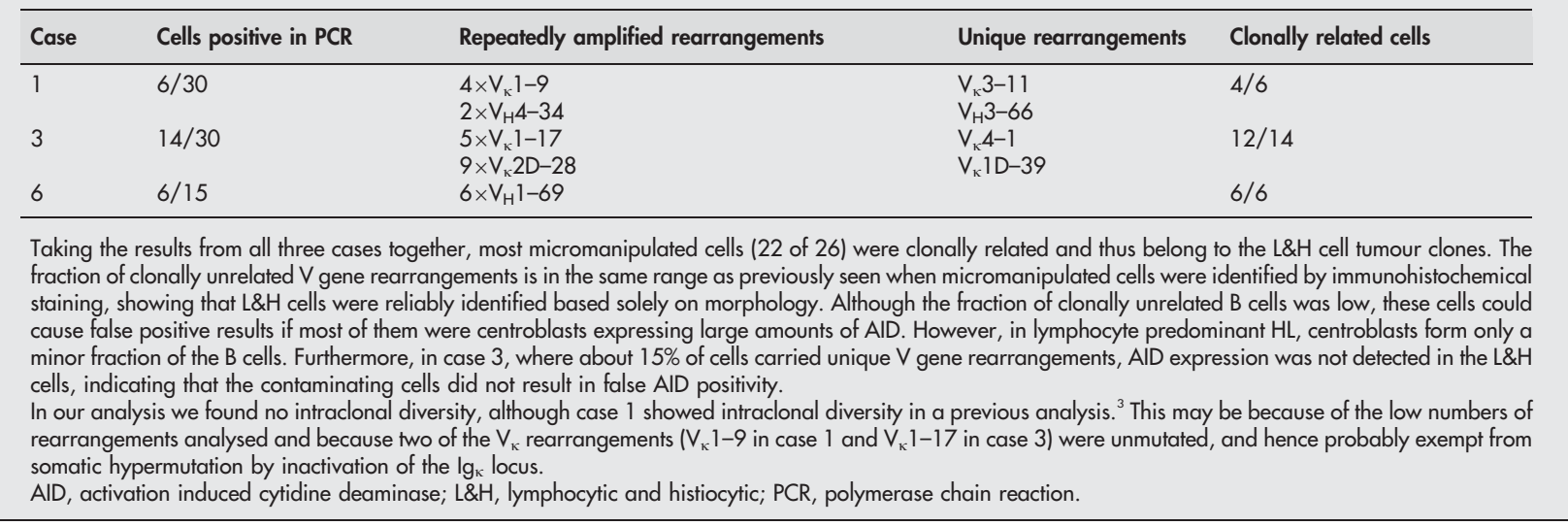

\section{Analysis of $\mathrm{V}$ gene rearrangements}

In three cases of lymphocyte predominant HL we performed single cell PCR to analyse $\mathrm{V}$ gene rearrangements. The staining procedures were the same as described above. L\&H cells were cut with the laser beam, isolated with a hydraulic micromanipulator, and subjected to PCR for V gene rearrangements at the IgH (immunoglobulin heavy chain), $\operatorname{Ig} \kappa$, and $\operatorname{Ig} \lambda$ ( $\kappa$ and $\lambda$ light chain) loci as described previously. ${ }^{2} 35$

\section{RESULTS}

L\&H cells for micromanipulation were selected on the basis of morphological criteria, and we performed $\mathrm{V}$ gene rearrangement analysis on single micromanipulated cells from three patients to confirm that the cells belonged to the tumour clones. This analysis revealed that most micromanipulated cells were clonally related (table 1). AID and 18S rRNA transcripts were measured using quantitative RT-PCR with Assays-on-Demand and RNA isolated from groups of 50 ( $\mathrm{L} \& \mathrm{H}$ cells and centroblasts) or 250 (endothelial cells as negative controls) micromanipulated cells. For each case and type of cell four samples were analysed and average $\mathrm{Ct}$ values (threshold cycle-the number of PCR cycles when the reporter fluorescence reaches a value that is significantly above background fluorescence for a specific PCR assay) were calculated. The $\mathrm{Ct}$ values for $18 \mathrm{~S}$ rRNA as an endogenous control confirmed that PCR with RNA isolated from 50 cells was consistently successful, and the average $\mathrm{Ct}$ values varied only within a range of three cycles within each group of samples (table 2).

The average $\Delta \mathrm{Ct}$ values for AID (Ct of AID - Ct of $18 \mathrm{~S}$ rRNA) in centroblasts were around 10 cycles, with only minor variations (9.3-11.9 cycles) between the three cases, whereas endothelial cells from the lymphadenites negative controls had $\Delta \mathrm{Ct}$ values between 22.6 and 29 cycles. The negative controls from the lymphocyte predominant HL cases had $\Delta \mathrm{Ct}$ values within a similar range (24.6-30.3 cycles). For the L\&H samples, there were large variations in the $\Delta \mathrm{Ct}$ values for AID, which ranged from 14.3 to 25.9 cycles, and only those obtained from cases 1,4 , and 6 (14.3, 16.8, and 20.1 cycles, respectively) were indicative of AID transcript expression above background. However, these values were clearly higher than those seen for centroblasts and indicate that AID expression in L\&H cells is lower than that seen in

Table 2 Quantitative real time RT-PCR for AID and 18S rRNA of micromanipulated centroblasts and L\&H cells

\begin{tabular}{|c|c|c|c|c|c|c|c|}
\hline Sample & & Ct 185 rRNA & Range & Ct AID & Range & $\Delta \mathrm{Ct}_{t}$ & Range \\
\hline \multirow[t]{6}{*}{ L\&H cells } & Case 1 & 21.3 & $9.8-23.3$ & 35.7 & $33.8-38.6$ & 14.3 & $11.3-17.6$ \\
\hline & Case 2 & 19.5 & $18.3-20.3$ & 41.5 & $37.9-50$ & 22.0 & $18.3-30.2$ \\
\hline & Case 3 & 20.2 & $19.3-21.9$ & 46.1 & $42.1-50$ & 25.9 & $20.4-30.5$ \\
\hline & Case 4 & 18.8 & $18.2-19.1$ & 35.6 & $34.8-37$ & 16.8 & $15.8-18.1$ \\
\hline & Case 5 & 20.6 & $17.6-22.6$ & 43.1 & $40-50$ & 22.6 & $18.9-27.4$ \\
\hline & Case 6 & 18.6 & $16.8-19.8$ & 38.6 & $35.9-41$ & 20.1 & $17.3-22.0$ \\
\hline \multirow[t]{3}{*}{ Centroblasts } & LN 1 & 24.6 & $23.6-25.6$ & 34.8 & $33.7-36.2$ & 10.3 & $8.4-12.6$ \\
\hline & LN 2 & 27.3 & $24.8-29$ & 36.6 & $34.7-38.2$ & 9.3 & $9.1-9.9$ \\
\hline & LN 3 & 25.4 & $23.7-28.2$ & 37.3 & $35.9-38.6$ & 11.9 & $10.3-14.9$ \\
\hline \multirow[t]{9}{*}{ Negative controls } & Case 1 & 18.9 & $17.5-20.3$ & 47.3 & $39.1-50$ & 28.4 & $20.8-32.5$ \\
\hline & Case 2 & 17.2 & $16.9-17.7$ & 41.9 & $38.3-50$ & 24.6 & $21.1-32.3$ \\
\hline & Case 3 & 18 & $16.8-19.2$ & 48.3 & $43.15-50$ & 30.3 & $24.9-33.2$ \\
\hline & Case 4 & 18.6 & $17.8-19.6$ & 45.4 & $40.32-50$ & 26.8 & $21.7-31.6$ \\
\hline & Case 5 & 20.3 & $19.3-21.3$ & 50 & $50-50$ & 29.7 & $28.7-30.7$ \\
\hline & Case 6 & 18 & $16.8-19$ & 47.3 & $39.4-50$ & 29.4 & $21.8-33.2$ \\
\hline & LN 1 & 23.2 & $21.9-25.4$ & 50 & $50-50$ & 26.8 & $24.6-28.2$ \\
\hline & LN 2 & 23.3 & $21.7-24.0$ & 45.9 & $40.9-50$ & 22.6 & $17.2-28.3$ \\
\hline & LN 3 & 20.2 & $18.4-21.7$ & 49.2 & $47-50$ & 29.0 & $26.4-31.6$ \\
\hline
\end{tabular}

The values are averages of four samples, and the highest and lowest values are shown as the range of Ct values. In addition to the results shown, for each case negative controls (water) were analysed in parallel and were consistently negative. In cases where AID transcripts were not detected, a Ct value of 50 was used for calculations, because a 50 cycle PCR was performed (a Ct of 50 cycles was seen for the L\&H cells of case 2 once, case 3 twice, and case 5 once, and for the negative controls of case 1 three times, case 2 once, case 3 three times, case 4 twice, case 5 four times, case 6 three times, LN1 four times, LN2 twice, and LN3 three times).

AID, activation induced cytidine deaminase; Ct, threshold cycle; L\&H, lymphocytic and histiocytic; LN, lymph node; RT-PCR, reverse transcription polymerase chain reaction. 


\section{Take home messages}

- We analysed whether activation induced cytidine deaminase (AID) is expressed in lymphocytic and histiocytic (L\&H) cells as an indicator of active somatic hypermutation in Hodgkin lymphoma (LH) tumour cells

- Transcription of AID was higher than background in three of six lymphocyte predominant $\mathrm{HL}$ cases, although it was lower than that seen in centroblasts

- Somatic hypermutation may remain active in L\&H cells in a considerable proportion of cases, increasing the risk of acquiring further transforming mutations

centroblasts. Whether the L\&H cells in the other cases did not express AID, or expression was below the sensitivity of our assay, or only a fraction of the lymphoma cells expressed AID remains unclear.

\section{DISCUSSION}

Taken together, we found that the AID transcription level was significantly higher than background, but below that seen in centroblasts, in a proportion of lymphocyte predominant HL cases, and that this expression pattern resembles that seen in other GC derived B cell non-HLs, such as follicular lymphoma, Burkitt lymphoma, and GC-type diffuse large B cell lymphoma, where significant amounts of AID transcripts were also found only in a proportion of the cases..$^{78}$ The detection of AID in half of the cases analysed suggests that somatic hypermutation may remain active in the established L\&H clone in at least some cases of lymphocyte predominant HL. Because AID activity has been associated with an increased risk of acquiring transforming events, ${ }^{8}$ the sustained expression of AID in L\&H cells may affect the risk of accumulating additional transforming mutations that play a role in transforming lymphocyte predominant HL into diffuse large cell lymphoma.

\section{ACKNOWLEDGEMENTS}

We thank V Distler for help with the laser micromanipulator and $\mathrm{R}$ Küppers for discussions and critical reading of the manuscript.

\section{Authors' affiliations}

A Mottok, M-L Hansmann, A Bräuninger, Department of Pathology, University of Frankfurt, Theodor-Stern-Kai 7, 60590 Frankfurt, Germany

Correspondence to: Dr A Bräuninger, Department of Pathology, University of Frankfurt, Theodor-Stern-Kai 7, 60590 Frankfurt,

Germany; braeuninger@em.uni-frankfurt.de

Accepted for publication 18 February 2005

\section{REFERENCES}

1 Hansmann ML, Weiss LM, Stein H, et al. Pathology of lymphocyte predominance Hodgkin's disease. In: Mauch PM, Armitage JO, Diehl V, et al, eds. Hodgkin's disease. Philadelphia: Lippincott Williams Wilkins, 1999: 168-90.

2 Küppers R, Rajewsky K, Zhao M, et al. Hodgkin disease: Hodgkin and ReedSternberg cells picked from histological sections show clonal immunoglobulin gene rearrangements and appear to be derived from $B$ cells at various stages of development. Proc Nat Acad Sci U S A 1994;91:10962-6.

3 Braeuninger A, Küppers R, Strickler JG, et al. Hodgkin and Reed-Sternberg cells in lymphocyte predominant Hodgkin disease represent clonal populations of germinal center-derived tumor B cells. Proc Natl Acad Sci U S A 1997; $94: 9337-42$

4 Marafioti T, Hummel M, Anagnostopoulos I, et al. Origin of nodular lymphocyte-predominant Hodgkin's disease from a clonal expansion of highly mutated germinal-center B cells. N Engl J Med 1997;337:453-8.

5 Klein $U$, Goossens $T$, Fischer $M$, et al. Somatic hypermutation in normal and transformed human B cells. Immunol Rev 1998;162:261-80.

6 Muramatsu M, Kinoshita K, Fagarasan S, et al. Class switch recombination and hypermutation require activation-induced cytidine deaminase (AID), a potential RNA editing enzyme. Cell 2000;102:553-63.

7 Pasqualucci L, Guglielmino R, Houldsworth J, et al. Expression of the AID protein in normal and neoplastic B cells. Blood 2004; 104:3318-25.

8 Smit LA, Bende RJ, Aten J, et al. Expression of activation-induced cytidine deaminase is confined to B-cell non-Hodgkin's lymphomas of germinal-center phenotype. Cancer Res 2003;63:3894-8.

9 Lossos IS, Levy R, Alizadeh AA. AID is expressed in germinal center B-cell-like and activated $B$-cell-like diffuse large-cell lymphomas and is not correlated with intraclonal heterogeneity. Leukemia 2004;18:1775-9.

10 Forconi F, Sahota SS, Raspadori D, et al. Hairy cell leukemia: at the crossroad of somatic mutation and isotype switch. Blood 2004;104:3312-17.

11 Aarts WM, Bende RJ, Steenbergen EJ, et al. Variable heavy chain gene analysis of follicular lymphomas: correlation between heavy chain isotype expression and somatic mutation load. Blood 2000;95:2922-9. 